\title{
Visual cortical prosthesis : an electrical perspective
}

\author{
Léo Pio-Lopez ${ }^{1}$, Romanos Poulkouras ${ }^{2,3}$, and Damien Depannemaecker ${ }^{4}$ \\ ${ }^{1}$ Independent researcher, 13006, Marseille, France \\ ${ }^{2}$ Department of Bioelectronics, Ecole Nationale Supérieure des Mines, CMP-EMSE, 13541 Gardanne, \\ France \\ ${ }^{3}$ Institut de Neurosciences de la Timone, UMR 7289, CNRS, Aix-Marseille Université, Marseille, France \\ ${ }^{4}$ Department of Integrative and Computational Neuroscience, Paris-Saclay Institute of Neuroscience, \\ Centre National de la Recherche Scientifique, France
}

\begin{abstract}
The electrical stimulation of the visual cortices has the potential to restore vision to blind individuals. Until now, the results of visual cortical prosthetics has been limited as no prosthesis has restored a full working vision but the field has shown a renewed interest these last years thanks to wireless and technological advances. However, several scientific and technical challenges are still open in order to achieve the therapeutic benefit expected by these new devices. One of the main challenges is the electrical stimulation of the brain itself. In this review, we analyze the results in electrode-based visual cortical prosthetics from the electrical point of view. We first briefly describe what is known about the electrode-tissue interface and safety of electrical stimulation. Then we focus on the psychophysics of prosthetic vision and the state-of-the-art on the interplay between the electrical stimulation of the visual cortex and phosphene perception. Lastly, we discuss the challenges and perspectives of visual cortex electrical stimulation and electrode array design to develop the new generation implantable cortical visual prostheses.
\end{abstract}

Keywords - visual cortical prosthesis, brain-machine interface, electrical stimulation, prosthetic vision

\section{Introduction}

In 2019, according to the World Health Organization, there were 1 billion people blind and moderately-to-severely visually impaired in the world. It is predicted that this number will increase significantly in the next decades due to the ageing of the population [67]. Visual deficit can therefore impose a huge social and economic burden. The causes of visual deficits are multiple. It can be due to pathologies of the retina as retinis pigmentosa, age-related macular degeneration, optic nerve, retina traumatisms due to accidents or even tumors surgery [25]. The nature of these last pathologies and traumatisms, destroying the neurons of the retina or the fibers of the optic nerve leads to the conclusion that we can't only rely on retinal prosthetics to treat and restore vision. The visual cortex remains generally intact in these different kinds of visual deficits opening the way to the field of visual cortical prosthesis for treatment.

At the origin of the field of the visual prosthetics, there is the experimental observation reporting the perception of a flash of light called phosphenes in the visual field, induced by electrical stimulation of the visual streams. This has been demonstrated for the visual cortex by 38. Since this seminal work, different ways of inducing these phosphenes have been developed, in particular through implanted electrodes. The core idea of visual prosthetics is to produce a pixelized vision made of multiple phosphenes induced by electrical stimulation of the visual streams. We can now categorize the main stream of visual cortical prosthetics field according to the used type of electrodes: surface electrodes (subdural electrodes) and intracortical electrodes. We can consider then that the field cortical visual prosthetics with surface electrodes began with 
the work of Brindley and Lewin study [12. They showed that a blind, 52 years old patient, could perceive phosphenes by electrical stimulation of the visual cortex. This first line of research has been then followed by the Dobelle group [29, 28, 31, 30.

From the nineties, the field see the development of the visual cortical prosthesis with intracortical electrodes. Schmidt and colleagues showed that a phosphene can be induced by electrical stimulation of the visual cortex with microelectrodes penetrating the cortex. This second line of research is now more predominantly followed [89, 10, 64.

But despite these advances in phosphene induction, few visual cortical prosthetis projects have emerged during this period. However, the recent publication of reviews on cortical visual prosthetics emphasize a renewed interest in this topic [5, 63, 64]. Indeed, new advancements in electrodes design and wireless power opened new opportunities. Nevertheless, at present, no cortical prosthesis has restored a useful vision (navigation, reading, object and facial recognition). This therefore represents an important issue and several challenges remain to be overcome.

In this article, we adopt an electrical perspective on the field of visual cortical prosthetics. We first describe the state-of-the-art about the electrode-tissue interface and safety of electrical stimulation. Then we focus on the psychophysics of prosthetic vision and the state-of-the-art on the interplay between the electrical stimulation of the visual cortex and phosphene perception. Lastly, we discuss the challenges and perspectives of visual cortex electrical stimulation and electrode array design for the development of the new generation of visual cortical prostheses.

\section{Principles of electrical stimulation of the brain}

The electrodes produce an effect on the tissue at short time scales (from millisecond to minutes) during stimulation, depending on the electrical parameters. At longer time scales (hours-years), the electrode effect on the tissue depends on frequency of recurrent stimulation and on the electrode material creating encapsulation of electrodes due to glial reaction, tissue inflammation or neuronal loss [6, 72]. The reaction of biological tissue is complex and the inflammatory response may affect properties of the interface, by keeping the same electrical parameters it may change the effects of the stimulation. The factors influencing the efficiency of electrical neural stimulation seem simple, but they are not completely understood.

The principal focus of electrical stimulation is on the interface between the electrode and the neural tissue. Electrochemistry mechanisms have been extensively studied and described [57, 32. Charge is carried by electrons in the implant material, and then by ions in the neural tissue. The effect of the electrode on the tissue can be capacitive, where no electrons are transferred from the electrode material to the tissue, or Faradaic, where electrons are transferred from the electrode material to the electrolyte, resulting in the oxidation or reduction of different chemical species in the neural tissue [77. Ideally, stimulation should remain within the capacitive domain at all times to minimise the risk of damage to the nervous system. Chemical species produced by Faradaic reactions can either be toxic for neurons, or damage them indirectly by decreasing their ability to compensate for toxic substances, and by increasing their sensitivity to other stress factors [23. The electrolysis of water may appear with a potential of only 1.23 Volt. The corresponding chemical reactions are the following:

$$
\begin{gathered}
2 \mathrm{H}_{2} \mathrm{O}+2 \mathrm{e}^{-} \longrightarrow \mathrm{H}_{2}+2 \mathrm{OH}^{-} \\
\mathrm{O}_{2}+\mathrm{H}_{2} \mathrm{O}+2 \mathrm{e}^{-} \longrightarrow \mathrm{OH}^{-}+\mathrm{O}_{2} \mathrm{H}
\end{gathered}
$$

It leads to a $\mathrm{pH}$ modification and may damage the tissue. The $\mathrm{pH}$ alteration is associated with pathological brain activities such as spreading depression or seizures [61, 74].

Most electrical stimulation of the nervous system consists of biphasic pulses. The first (usually cathodic phase) of the biphasic pulse is used to depolarise the neural membranes in the surrounding tissue and initiate the desired action potentials. Following that, a balanced anodic phase is used 
to reverse any chemical reactions that might have taken place during the first phase. A brief pause between the cathodic and anodic phase allows the electrode to discharge completely and return to potential equilibrium 22 .

Stimulation systems used for experimental or clinical electrophysiology are either current- or voltage- driven. In the first case, a given current value is injected by the stimulator applying the necessary electric potential, while in the second, the electric potential is set and controlled by the user while the device injects the corresponding current. In general, current-driven stimulation is more reliable as it allows a control over the current injected. However, for long-term applications, it is more power consuming [76, 52. Current-driven stimulation also allows to know the range of the injected charges offering a better safety criterion.

Indeed, Shannon demonstrated that one of the essential parameters for safe stimulation is the charge injected per pulse phase [85]. This is deduced by the current level times the duration of the pulse $Q=$ I.t. For constant current stimulation, charge can be easily derived. However, in the case of constant voltage stimulation, the current does not remain stable, allowing only for an approximation of the injected charge per phase. These authors have demonstrated that longer pulse widths require higher charge content to elicit a response, even if their absolute current value may be lower than in the case of shorter pulses [85]. Therefore shorter pulses with minimal amplitude should be favoured to move the injected charge per phase value as far away as from the area of parametric space that can be harmful to the tissue.

In monopolar stimulation, the current flows between the stimulating electrode and the ground, usually placed away from the electrodes. The resulting electric field is diffuse. Bipolar stimulation is between two electrodes in the array, producing a more densely-packed electric field. The choice between the two largely depends on the position of the target neurons relative to the two electrode tips for bipolar stimulation. If the target is placed in between two electrode tips, bipolar stimulation may be preferred, as the generated electrical field is more focal. The decision is largely patient specific in clinical settings, such as in deep brain stimulation [43, 87.

In all cases, the applied voltage values (either through constant voltage stimulation, or as the voltage required to drive set current in constant current stimulation), should remain below the potential levels where irreversible electrochemical reactions begin to occur either in the electrolyte or the electrode surface. Otherwise, this would lead to tissue damage and electrode corrosion respectively. The interested reader can refer to the review of Merrill and colleagues [60].

The material of the electrodes has also an impact on the parameters and safety of electrical stimulation. A major advance in this area is the introduction of PEDOT and PEDOT:PSS electrodes. This kind of electrode presents electrochemical stability, high conductivity and impedance reduction [26, 73].

\section{Psychophysics of prosthetic vision}

This field explores the design and visual information processing required for the visual tasks necessary in daily life, like reading, navigation, or the recognition of objects or faces under prosthetic vision (for a review, see Chen [20, 21]). This line of research is crucial for electrode array design as its results can set constraints on the number, configuration, interspace and placement of electrodes on the visual cortex. And ultimately, the choice of these parameters has implications on electrical stimulations needed to restore a useful vision as psychophysics can shed light on the need of simultaneous electrode stimulation, time of stimulation etc...

Traditionally, in the studies of prosthetic vision, a video camera is on the head of the subjects recording the visual scene and sending them the visual information on a monitor through a pixelized filter simulating prosthetic vision for different tasks [18, 20, 21]. Cha and colleagues studied the optimal number of electrodes for reading. Their experiment estimated the number of pixels to 600 to restore a vision allowing reading. This minimal number was then increased to 625 (array of electrodes of 25x25) by Cha and colleagues [18]. Sommerhalder [86] reduced this optimal number and showed that 300 electrodes in foveal vision were necessary to reach a quasi-perfect 
reading of isolated words (almost $90 \%$ of words read correctly). They also studied reading in semi-peripheral vision ( $15^{\circ}$ of visual angle) and reported that it is also possible after a period of learning of a month during one hour a day (64-85\% of correct reading) with 300 electrodes. This is important given the foveal projection of the visual field on the cortex is in the calcarine sulcus and is therefore more difficult to access [101. Indeed, if we can't reach it, it would be possible to use electrodes in the projection of the semi-peripheral vision on the cortex. Reading with prosthetic vision is also possible with a smaller number of electrodes. Fu et al. showed that with only a low resolution of 36 (array of $6 \times 6$ ) and 64 phosphenes (array 8x8), reading was also possible but it will strongly affect reading speed [40].

For facial recognition, Vurro et al. showed that 100 electrodes allow a facial recognition included between $70-80 \%$ of recognition rates with various configurations of electrodes (squared, hexagonal, log-polar) with an advantage however for the hexagonal after a pre-treatment of the image [100. Other results also show that a hexagonal matrix would be more successful for the prosthetic vision [19]. More recently, a study suggests that facial recognition requires much more phosphenes than previously reported and at least an array of 32x32 electrodes is needed 44 .

In terms of functional performance, a low-resolution electrodes array would allow wayfinding with a 6 x10 array 98 . For complex scene recognition, more electrodes are needed, Zhao and colleagues suggested at least $48 \times 48$ electrodes for complete recognition would be necessary [107.

In any cases, phosphene density is the main determinant of visual acuity and a low-resolution visual prosthesis would lead to low efficiency on most acuity mediated visual tasks such as reading, object identification, face recognition, obstacle avoidance, etc... [18]. Therefore, a visual cortical prosthesis able to restore a useful general vision in everyday life will likely have hundreds of electrodes inducing phosphenes in the visual field of the patient.

\section{Phosphene perception and electrical stimulation}

\subsection{Visual cortical prosthesis and surface electrodes}

This first line of research has been followed by the groups of Brindley [11, 80] and Dobelle $[29,28,31,30]$. The pioneering work of Brindley [11] showed that the common evoked phosphene is described as a flash of white light, as 'a star in the sky' or having 'the size of a grain of sago at arm's length' and that the phosphene perception change and stretch with the distance from the foveal projection on the cortex [11]. Most of the projects who involved a clinical development have been based on surface electrodes. The advantage of this approach is the minimal invasivity of the electrodes on the cortex.

The brain-machine interface is generally made of platinum electrodes $[11,29,28,31$, 30], with squared [11], or circulars shapes $[28,31,30]$. The array is set on subdural configuration and the electrode arrangement of the electrodes themselves is squared [11], or hexagonal [28, 31, 30]. The minimal electrode interspace to obtain two distinct phosphenes varies according to the studies. Indeed, it is about $2.4 \mathrm{~mm}$ for Brindley first study [11], 1.5 to $3 \mathrm{~mm}$ for Dobelle and Mladejovsky [29], 1.5-2 $\mathrm{mm}$ for [70] and until $8 \mathrm{~mm}$ for Pollen and colleagues [71. The minimal interspace would vary with the localization, Pollen suggests that this surprising results compared to those of Dobelle and Brindley would be due to the placement of his electrodes in the foveal vision where a large portion of cortex is used to analyze a small region of the visual field [71]. The size of the electrodes would not influence the phosphene perception [28]. But Pollen reported that it was possible to evoke phosphene with electrodes having a diameter of $0.25 \mathrm{~mm}$ and that the evoked phosphene are perceived as smaller than those obtained with electrodes of a diameter of $0.5 \mathrm{~mm}$ [70].

Winawer and colleagues reported that phosphene size increases with eccentricity and amount of charge but the area activated increases with the amount of charge but not with eccentricity [106] which is in line with non-human primate studies with microelectrodes [91, 92, 89]. Bosking et al. also found with human subjects that the size of the phosphene depends on the amplitude 


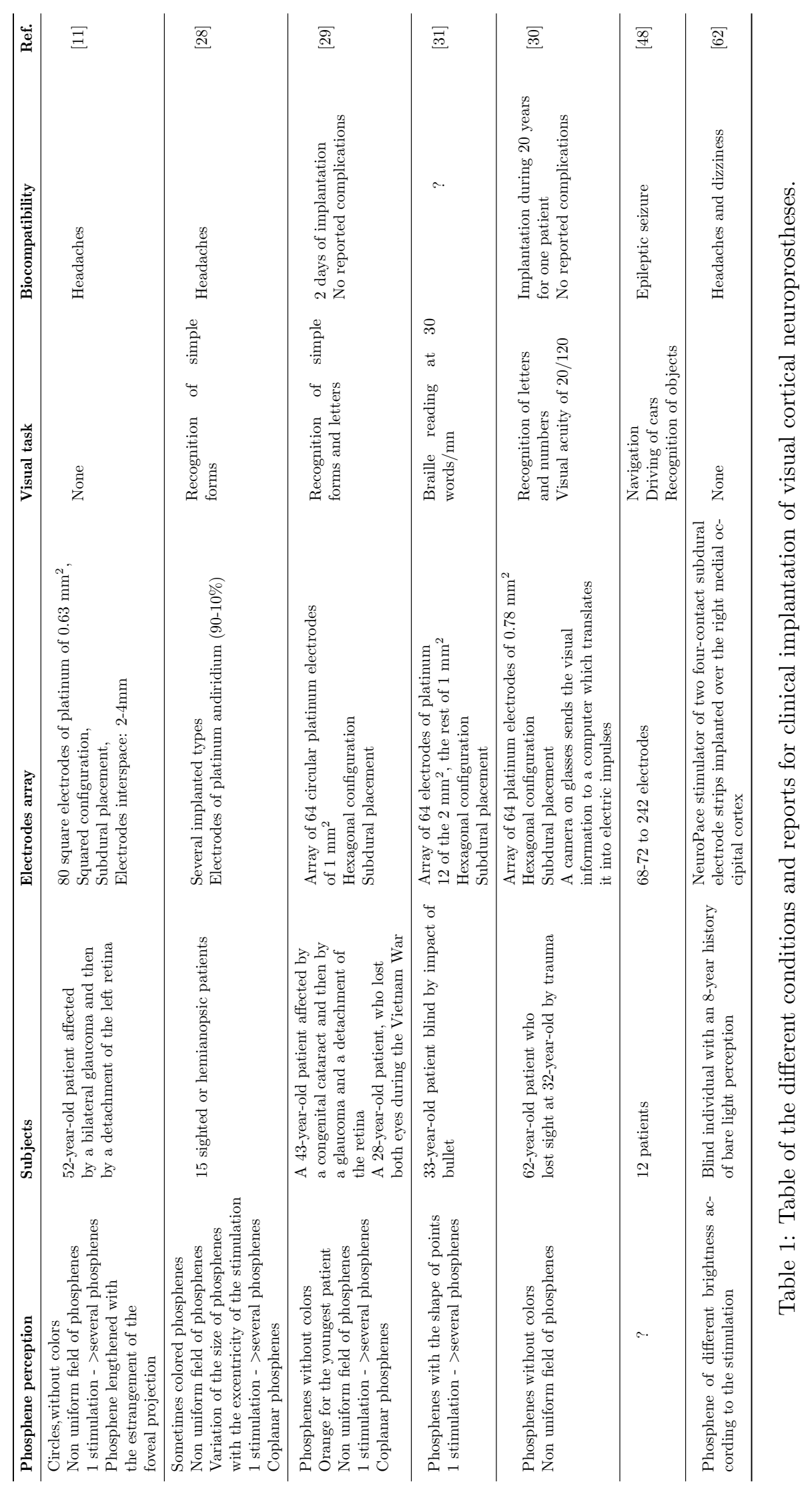




\begin{tabular}{|c|c|c|c|c|c|c|c|}
\hline Pulse Waveform & Polarity & Amplitude & Frequency & Pulse duration & $\begin{array}{c}\text { Train } \\
\text { duration }\end{array}$ & Impedance & Ref. \\
\hline$?$ & $\begin{array}{l}\text { Monophasic } \\
(-)\end{array}$ & 90 mW (mean power) & $100 \mathrm{~Hz}$ & $200 \mu \mathrm{s}$ & $?$ & $3000 \Omega$ & {$[11]$} \\
\hline Symmetrical & $\begin{array}{c}\text { Monophasic } \\
(+,-) \\
\text { Biphasic } \\
(+/-,-/+) \\
\end{array}$ & $1-5 \mathrm{~mA}$ & 30 to $200 \mathrm{~Hz}$ & 0.25 to $2 \mathrm{~ms}$ & 5 to 15 pulses & $3000 \Omega$ & {$[28]$} \\
\hline Symmetrical & $\begin{array}{c}\text { Biphasic } \\
(+/-)\end{array}$ & $0.6-9.3 \mathrm{~mA}$ & $50 \mathrm{~Hz}$ & $0.5 \mathrm{~ms}$ & $>3 \mathrm{~s}$ & $?$ & {$[29]$} \\
\hline Squared & $\begin{array}{c}\text { Biphasic } \\
(-/+)\end{array}$ & $0.5-3 \mathrm{~mA}$ & $50 \mathrm{~Hz}$ & $0.25 \mathrm{~ms}$ & $0.1-1 \mathrm{~ms}$ & $?$ & {$[31]$} \\
\hline Symmetrical & $\begin{array}{c}\text { Biphasic } \\
(-/+)\end{array}$ & $10-20 \mathrm{~V}$ & $30 \mathrm{~Hz}$ & $500 \mu \mathrm{s} /$ phase & $\begin{array}{c}\text { Train of } 6 \\
\text { pulses }\end{array}$ & $?$ & {$[30]$} \\
\hline Squared & Biphasic & $0.2-5 \mathrm{~mA}$ & $5-100 \mathrm{~Hz}$ & $200-1,000 \mu \mathrm{s}$ & $0.2-1 \mathrm{~s}$ & $?$ & {$[106]$} \\
\hline Squared & $\begin{array}{c}\text { Biphasic } \\
(-/+)\end{array}$ & $0.3-4 \mathrm{~mA}$ & $200 \mathrm{~Hz}$ & $0.1 \mathrm{~ms}$ & $200-300 \mathrm{~ms}$ & $?$ & 7 \\
\hline$?$ & $\begin{array}{c}\text { Monophasic } \\
\text { Biphasic } \\
(-/+) \\
\end{array}$ & $0.3-7.5 \mathrm{~mA}$ & $200 \mathrm{~Hz}$ & $0.1 \mathrm{~ms}$ & $200-300 \mathrm{~ms}$ & $?$ & 3] \\
\hline
\end{tabular}

Table 2: Electrical parameters of the stimulation of the human visual cortex to induce phosphenes with surface electrodes. The parameters refer to the common satisfactory parameters as reported in the articles.

of the stimulation. In addition, they discovered that phosphene size saturates at a relatively low current levels (around $2 \mathrm{~mA}$ ) 9]. Various works also show a relation between the amplitude of the pulse and the luminosity of the phosphene [29, 30] although this relationship was observed for a study as non-linear [28]. It also seems that a frequency of stimulation superior to $30 \mathrm{~Hz}$ stop the flickering of the phosphene and that the reversal of polarity of pulses $(+/-,-/+)$ has no influence on the perception of the phosphene [29]. Another point is the continuation of the phosphene after the interruption of the stimulus [11, 29]. According to Bak and colleagues [1], this obstinacy would be due to epileptic discharges such as reported by Pollen [71]. The minimum thresholds of stimulation to evoke a phosphene is between $0.2-4 \mathrm{~mA}$ depending on the electrodes and the most common stimulation reported in the studies is biphasic and symmetric (see Table 2]. Shape and localization seem to stay globally stable over time [80, 62] and multiple induced phosphenes are coplanar [29].

One main challenge of visual cortical prosthetics is the specificity of the stimulation, e.g. for one stimulation, one phosphene. Indeed, the phosphene induction by electrical stimulation with surface electrodes has been reported sometimes as non-specific [11, 80, 29, 28, 31, 30]. An interaction between two adjacent phosphenes is also possible appearing with the shape of a bright cloud [13. Beauchamp and colleagues also showed that when multiple electrodes were stimulated simultaneously, phosphenes could fuse into larger visual perceptions, not allowing independent recognition of the induced phosphenes [3]. However, more recent studies have shown reliable specific induction of phosphenes with surface electrodes separated by 6-10 mm [8]. They also could stimulate 4-6 electrodes and induce up to 5 phosphenes at the same time [8]. Therefore, it seems that producing a specific pattern using surface electrodes for different visual tasks is feasible and has been achieved for different cases (see Table 1).

Various visual tasks were achieved with these prostheses with surface electrodes. The first studies show that the recognition of letters and simple forms is possible $[31,30]$. The last implantation of the team of Dobelle would have allowed the recognition of objects, wayfinding and the driving of a car on a private parking lot by a non-assisted patient [48].

Nowadays, the research on the visual cortical prosthesis with surface electrodes seems only conducted by SecondSight Medical Products with the Orion device [63]. The main arguments advanced against the use of the electrodes of surface are the currents used in the milliampere 


\begin{tabular}{|c|c|c|c|c|c|c|c|}
\hline $\begin{array}{c}\text { Pulse } \\
\text { Waveform }\end{array}$ & Polarity & Amplitude & Frequency & $\begin{array}{c}\text { Pulse } \\
\text { duration }\end{array}$ & Train duration & Impedance & Ref. \\
\hline Squared & $\begin{array}{c}\text { Biphasic }(+/-, \\
-/+)\end{array}$ & $1.9-80 \mu \mathrm{A}$ & $200 \mathrm{~Hz}$ & $200 \mu \mathrm{s}$ & $125 \mathrm{~ms}$ & $?$ & 84 \\
\hline $\begin{array}{c}\text { Symmetrical, } \\
\text { not squared }\end{array}$ & $\begin{array}{c}\text { Biphasic }(+, \\
-)\end{array}$ & $10-20 \mu \mathrm{A}$ & $200 \mathrm{~Hz}$ & $400 \mu \mathrm{s}$ & $\begin{array}{c}1 \mathrm{~s} \text { stimulus trial } \\
\text { with three } 200 \mathrm{~ms} \\
\text { pulse trains, with } \\
\text { an intertrain } \\
\text { interval of } 200 \mathrm{~ms}\end{array}$ & $?$ & 96] \\
\hline$?$ & Biphasic & $1-100 \mu \mathrm{A}$ (as reported in 35]) & $?$ & $?$ & $?$ & $?$ & 37 \\
\hline
\end{tabular}

Table 3: Electrical parameters of the stimulation of the human visual cortex to induce phosphenes with intracortical electrodes. The parameters refer to the common satisfactory parameters as reported in the articles.

order and the low phosphene resolution obtained with this technology because of the large size and interspace of the electodes at the surface of the cortex. Nevertheless, this technology presents several advantages. Indeed, it is the less invasive approach and it is compatible with the fabrication of arrays of flexible electrodes [99, 56. This type of array would ease for example the surgical implantation in the calcarine fissure where we can find the most of the retinotopic maps of the foveal vision in humans [101].

\subsection{Visual cortical prosthesis and intracortical electrodes}

Most of the current projects follow the intracortical electrodes approach for the development of a visual cortical prosthesis. We can cite the Intracortical Visual Prosthesis Project (ICVP) at the Illinois University of Technology [79, the Cortical visual prosthesis for the blind CORTIVIS supported by the European Commission [36] or the Gennaris bionic vision system developed by Monash Vision Group supported by the Australian Research Council [55]. Intracortical electrodes present several advantages: they allow to reduce the necessary current to evoke a phosphene by several orders of magnitude [88, 84] and it is possible to implant hundred of electrodes on a very small surface of the cortex ( $4 \mathrm{~mm}^{2}$ with CORTIVIS for example).

Schmidt and colleagues showed that such intracortical electrodes allowed the perception of phosphenes with a patient blind for 22 years after glaucoma [84]. The phosphene is described as having a round shape and of size ranging from 0.2 to $2^{\circ}$ of visual angle, without colors or blue, yellow, red but not green $[1,84]$. With this kind of penetrating microelectrodes, the luminance of the phosphene increases with the amplitude of pulses but experiments also reported a reduced size $[1,84]$. It seems that a non-linear relation exists between the amplitude of pulse and the size of the evoked phosphene. During the stimulation, the luminosity of the phosphene can also decrease through time, maybe exhibiting a saturation effect [84]. Similarly with the results obtained with the surface electrodes, when at least 3 stimulations were simultaneously applied, phosphenes seemed coplanar for the patient and one stimulation can induce several phosphenes [84]. The minimal electrodes interspace to obtain two different phosphenes is $500 \mu \mathrm{m}$, but microelectrodes spaced with $250 \mu \mathrm{m}$ typically did not elicit specific phosphenes [84]. The study of Schmidt et al. [84] on humans has been stopped prematurely because of complications [83]. A similar array of electrodes was then implanted in the macaque [10]. Five months later, the study has also been interrupted, the monkey became lethargic because of a reaction of the nerve tissue at the level of electrodes and thus because of problems of biocompatibility.

The team of Normann developed the Utah Electrode Array (UEA), which has been approved by the Food and Drug Administration (FDA) for long-term human studies 65, 66. This array is a silicon-based matrix of 10x10 penetrating microelectrodes. Electrodes are long of $1.5 \mathrm{~mm}$ and are spaced out of $400 \mu \mathrm{m}$. Their tips are covered with platinum to facilitate the injection of currents. The observations on biocompatibility associated with the presence of this type of matrix can vary from an absence of reaction to the development of a gliosis or fibrosis between the array of electrodes and the brain, movement of the array or even bleedings [65]. Fernandez 
and colleagues recently implanted the UEA in the occipital cortex of a 57-year-old person during a six-month period. They found that stimulation thresholds necessary to induce phosphenes were in the 1-100 $\mu \mathrm{A}$ range [35]. This threshold also depends on the depth of stimulation as reported by De Yoe and colleagues in monkeys [27. The lower threshold has been found in layers 2/3 and more precisely at the level of the frontier between layer 3 and $4 \mathrm{~A}$. Another low threshold is found in layer 5. Tehovnik and colleagues, by using a similar protocol, found the lower threshold in layer 5 at a depth of $1.6 \mathrm{~mm}$ under the surface of the cortex 93. These results are coherent with those obtained by Bartlett and Doty 2. The chronaxies of V1 neurons for the ocular saccades are also lower in the deep layer $(0.17 \mathrm{~ms})$ than in the superficial $(0.23 \mathrm{~ms})$ [0].

The electrical stimulation parameters leading to phosphene induction have not been well studied in humans. The electrical stimulation parameters with intracortical electrodes (as reported in Table 3) are symmetric and the amplitude range from 1 to $100 \mu \mathrm{A}$. The pulse duration when reported is between 200 and $400 \mu$ s and train duration range from $125 \mathrm{~ms}$ to $1 \mathrm{~s}$ (three $200 \mathrm{~ms}$ pulse trains, with a inter-train interval of $200 \mathrm{~ms}$ ).

The intracortical approach presents several advantages: current in the microampere order (see Table 3), and the possibility of increasing the spatial resolution on a small part of the visual field. The main inconvenience is probably the biocompatibility. It is the most invasive technology and it could act negatively with the vascularization of the cortex [24. In addition, damage can be also induced by mechanical micromovements between the pulsating neural tissue and the static implants 69]. Nevertheless, the studies on cats and monkeys seem to show a good reaction of tissues through time [54, 102, 96]. It was shown that the encapsulation of electrodes does not prevent systematically the efficiency of the stimulation [41, 105]. Another disadvantage is the non-soft character of the arrays of intracortical electrodes [79, [97, 36]. As a large part of the visual field map of central vision is located in the calcarine sulcus [101], it seems difficult to reach this zone of interest with such arrays. It could be limiting factor given the importance of the foveal vision in reading, recognition of faces and the vision of details. In addition, it is still not clear if the array of penetrating microelectrodes can induce a complex pattern of phosphenes. Unlike surface electrodes, we didn't find any studies reporting the analysis of simultaneous stimulation of several electrodes or the achievement of visual tasks with such a prosthesis.

\section{Electrical stimulation and electrode array design: challenges, potential solutions and perspectives}

The studies reporting implantation of a visual cortical prosthesis either with surface or intracortical electrodes allow us to define several important points which need further investigations.

V1 accessibility A large part of the the visual field map of central vision on the cortex lies in the calcarine sulcus [101]. So far, no project has planned to implant a prosthesis in this area [64]. It seems very unlikely that a non-soft electrodes array will be implanted in this sulcus given the difficulty of access. However, flexible electrodes array would be a potential solution and particularly useful in order to induce phosphene in foveal vision. Some prototypes have been already developed for intracortical electrodes [78] and surface electrodes [56]. Cortical arrays of electrodes fabricated on smooth, flexible and ultrathin materials like parylene provide better coverage of the target area by conforming completely to the shape of the cortex. The same advances can also improve penetrating electrodes. By significantly diminishing their diameter and fabricating them on a soft, thin substrate, those implants promise to cause minimal damage during implantation, and no chronic damage, since the mismatch between a rigid material and soft neural tissue is mitigated due to the soft nature of the implant [82, 49]. Nevertheless, the implantation in the calcarine sulcus could lead to other technological problems including wireless transmission of information and long-term stability of the prosthesis.

High-number of electrodes for restoring a useful vision In order to restore a useful vision, it will be likely necessary to induce hundreds of phosphenes to achieve basic tasks of everyday life as psychophysics of prosthetic vision seem to indicate (see section 3). At present, the studies reported 


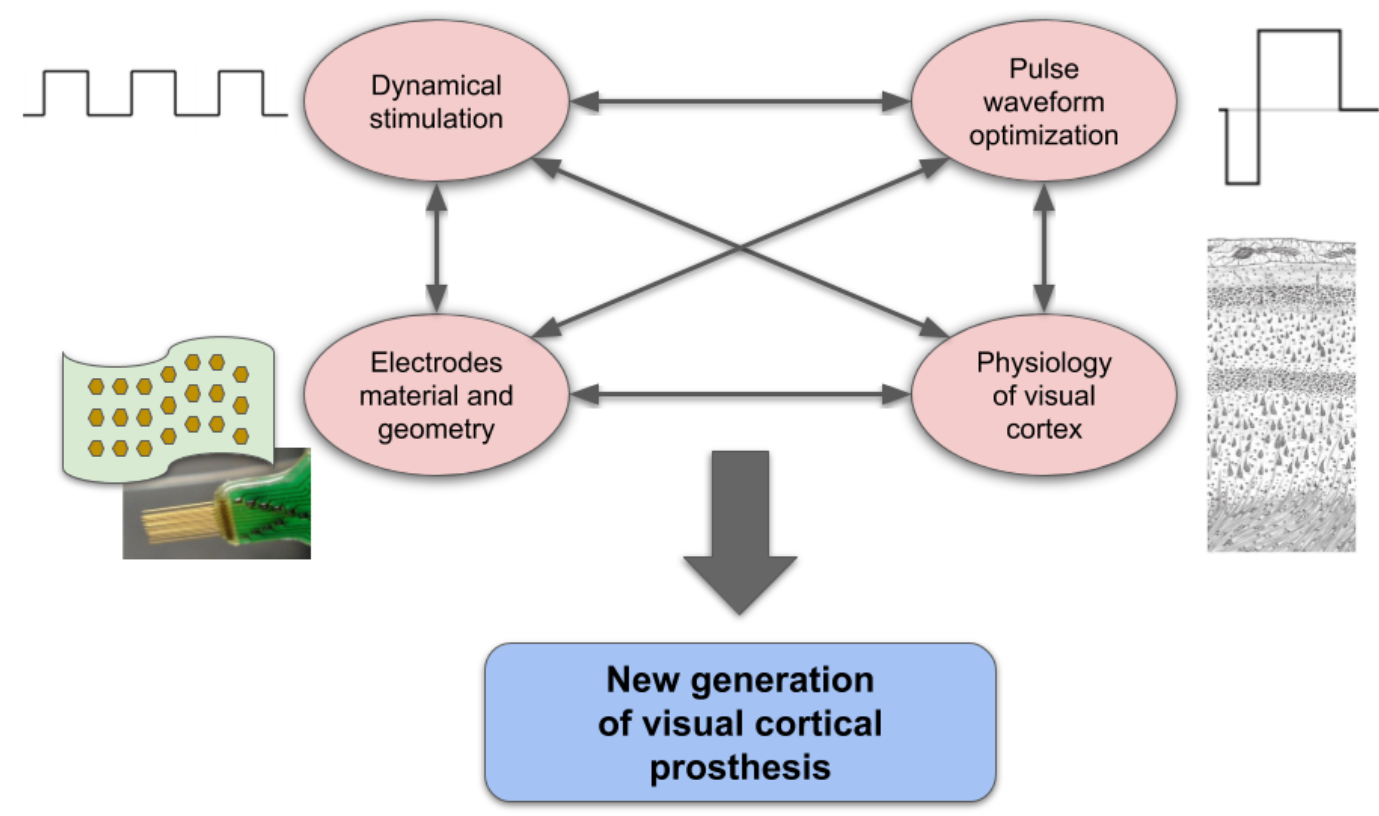

Figure 1: Interaction between different research fields leading to new generation of visual cortical prosthesis.

either development with surface/subdural electrodes or intracortical electrodes. No projects proposed a hybrid way. However, it presents an interesting benefit/cost ratio. Indeed, to minimize invasivity, intracortical electrodes could be implanted only in semi-peripheral vision and the foveal and peripheral vision would be supported by surface electrodes stimulation. The clinician would have the advantages of both techniques and to limit their inconveniences. Intracortical electrodes would allow increasing the spatial resolution in semi-peripheral vision due to their low size and the minimal interspace electrodes of $500 \mu \mathrm{m}$ to obtain distinct phosphenes. This technology would allow to reach a number of electrodes high enough for acuity-mediated tasks like reading, navigation or object and face recognition. In addition, the amount of current needed to induce the phosphenes will be reduced by the use of intracortical electrodes array while having a good mapping of the rest of the visual field with surface electrodes. The other way, surface electrodes would limit the invasivity of the prosthesis.

Waveform uniformity It seems difficult to conclude on the most adequate parameters of stimulation for the evocation of phosphene in the visual field of the patients: parameters are variable or incomplete between the various studies with surface or intracortical electrodes. More particularly, the shape of the electrical wave is squared or symmetrical in the majority of the studies (or not reported, see Tables 2 and 3). Squared waveforms are a common shape for electrical brain stimulation. But thresholds are similar for cells and axons with the rectangular cathodic stimulation [75, 45, 59. Different studies demonstrated that asymmetric waveforms can increase the selectivity of the stimulation by modifying the degree of inactivation of voltage-dependent sodium channels [81, 42. Imbalanced waveform can also mitigate the dissolution of platinum electrodes [50. This shows the importance of the relationship between the materials used, the electrode tissue interface and the waveform. This approach should be integrated for the development of the new generation of electrode-based visual cortical prosthesis.

Variability in frequency and duration of the pulses We can also observe a strong variability in the duration of the pulse (see Table 2 and 3). This lack of selectivity of the stimulation [29, 28] and the lack of clear data makes difficult the interpretation of the results concerning the optimal electrical parameters for evoked visual responses. The frequency and duration of the pulses can clearly be a criterion to stay in a safe stimulation domain while maximizing the perceptual effect 
for long term use. New approaches are also being developed in the field of deep brain stimulation like the technique of current steering. It consists of using multiple electrodes to control the flow of current through the neural tissue, thus allowing a level of fine control over the volume of neural tissue activated by the stimulation. While a novel technique, its combination with high density implants holds promise of improvement, as a higher number of electrodes allows for more options in the way the current can be steered. Such novelties in both stimulation approach and implant hardware could be combined to improve the selectivity of the stimulation for visual restoration 16. 4.

Shape of electrodes The shape of the electrode has two major influences. Firstly, the shape have an impact on the electrode performance and on energy consumption, which can be an important criterion for long term implant and neural stimulation efficiency required for clinical efficacy. The relation of perimeter to surface area have an influence on the spatial distribution of current density 104 and it has been shown for example that fractal-shaped microelectrodes superior have better charge injection capacity, despite having smaller perimeter than other designs [68.

In second, the shape of the electrode has a direct influence on the electrical properties of the stimulation [14. By changing the shape of electrodes, we can change the shape of the volume of activated neural tissue. Indeed, by segmenting the electrode, or, to change the shape of the contacts can increase the localization of current delivery into specific populations of neuron [103, 15]. A combination of these effects taking into account the spatial non-homogeneity of visual projection in the cortex could significantly improve the resolution of evoked visual perceptions.

Chronic stimulation The early studies report the implantation of cortical neuroprosthesis during years and even one during 20 years [30]. The reported complications are headaches and would be due to an activation of the meningeal fibers $[11,28]$. A reported epileptic seizure is also reported, probably induced by an over stimulation [48]. More recently, Niketeghad and colleagues implanted subdural electrodes (a NeuroPace stimulator) in a blind individual [62]. They don't report any serious adverse events except mild headaches and dizziness in three instances. Nevertheless, it is difficult to conclude on the parameters of safe chronic stimulation. Indeed, in addition to not having a clear view on the parameters of electrical stimulation applied in the early studies, no implantation of a fully working neuroprosthesis has been achieved, allowing the induction of hundred of phosphenes via the simultaneous activation of a large number of stimulation electrodes. Long-term stability The stability of the prosthesis for years is a challenge. Indeed, Towle and colleagues investigated the brain from a blind human subject who had a cortical visual prosthesis implanted for 36 years by Dobelle team [95]. They found a unexpected rotation of the electrode array ranging from 25 to 40 degrees away from the midsagittal plane. They suppose that the torque of the connecting cable could be the cause of this rotation. Wireless prosthesis is a potential solution to this problem [62]. Nevertheless, both approaches in cortical visual prosthetics are not equal in terms of stability. Intracortical microelectrodes are highly invasive. By penetrating the cortex, tissue responses like glial encapsulation, neuroinflammatory reponses or neuronal cell loss can prevent their long-term stability and efficiency [53, 6, 72]. Surface/subdural electrodes are now known to have more reliable long-term stability [94, 47].

Biocompatibility and electrode materials The biocompatibility of the materials used for the implantable part is essential for long-term implantation. In this case, the biocompatibility of a material is assessed by its ability not to induce toxicity, and to produce a minimal inflammatory reaction of the tissues for the long term use. Historically, the materials for implantable electrodes manufacturing are silicone and platinum, and have been well characterized in this context 33 . During the last decade, the development of new materials for electrodes such as the PEDOT:PSS (mixtures of poly (3, 4-ethylenedioxythiophene) and polystyrenesulfonate) has demonstrated progress in terms of biocompatibility [17. The improved conductivity and impedance of new materials like PEDOT:PSS has a direct positive impact in any kind of brain interface. The improved conductivity allows for electrodes to deliver electrical stimulation with a minimal risk of damage to tissue, while their low impedance provides a much better recording quality, therefore improving both monitoring and evoking neurophysiological activity in patients. PEDOT:PSS has the high conductivity among organic thermoelectric materials and increase charge-transfer 
efficiency [17]. The fact that these improved properties are intrinsic to the material paves the way for fabrication of scalable electrodes down to the micro-scale that can efficiently stimulate and record activity in a very localised and selective manner. In addition, these novel materials are compatible with flexible substrates and microfabrication techniques offers additional possibilities to improve current brain implants. Both surface and intracortical electrodes can be improved through these advances.

Micro-coils Another strategy outside the traditional electrode-based cortical visual prosthetics is the use of magnetic stimulation by means of micro-coils [51, 39. They are small implantable inductors that magnetically activate neurons. The brain stimulation of cortical pyramidal neurons in vitro has been reported as reliable and could be confined to spatially narrow regions $(<60 \mu \mathrm{m})$ [51]. Similarly to electrode stimulation, a phosphene can be induced by magnetic stimulation [58, 46] and therefore this strategy could lead to restoration of vision. Other strategies are possible as described in 34.

\section{Conclusion}

Cortical visual prosthetics spurred a renewed interest these last years thanks to wireless and technological advances. As we saw it, compared to the retina, the optical nerve or with the LGN, the visual cortex presents a large surface of stimulation and remain usually intact after visual impairments. In this study, we focused on the electrical stimulation of the visual cortices and related aspects. We showed that despite several developments, there is still a knowledge gap concerning the safe parameters of electrical stimulation for multi-phosphene induction. To fill this gap and reach a complete clinical efficiency of the electrode-based cortical visual prosthesis seems key for the future. The next generation of visual cortical prosthesis will come hand-to-hand with new technological development of electrodes (in terms of materials and geometry), and the extension of approaches based on temporal sequences and new waveforms of stimulation to increase the efficiency and safety of phosphene induction. 


\section{References}

[1] M Bak, JP Girvin, FT Hambrecht, CV Kufta, GE Loeb, and EM Schmidt. Visual sensations produced by intracortical microstimulation of the human occipital cortex. Medical and Biological Engineering and Computing, 28(3):257-259, 1990.

[2] JR Bartlett and RW Doty. An exploration of the ability of macaques to detect microstimulation of striate cortex. Acta Neurobiol Exp (Wars), 40(4):713-727, 1980.

[3] Michael S Beauchamp, Denise Oswalt, Ping Sun, Brett L Foster, John F Magnotti, Soroush Niketeghad, Nader Pouratian, William H Bosking, and Daniel Yoshor. Dynamic stimulation of visual cortex produces form vision in sighted and blind humans. Cell, 181(4):774-783, 2020.

[4] Christopher J. Bettinger, Melanie Ecker, Takashi Daniel Yoshida Kozai, George G. Malliaras, Ellis Meng, and Walter Voit. Recent advances in neural interfaces - materials chemistry to clinical translation. MRS Bulletin, 45(8):655-668, August 2020.

[5] Alexy Bhowmick and Shyamanta Hazarika. An insight into assistive technology for the visually impaired and blind people: state-of-the-art and future trends. Journal on Multimodal User Interfaces, 11:1-24, 012017.

[6] Roy Biran, David C Martin, and Patrick A Tresco. Neuronal cell loss accompanies the brain tissue response to chronically implanted silicon microelectrode arrays. Experimental neurology, 195(1):115-126, 2005.

[7] W. H. Bosking, P. Sun, M. Ozker, X. Pei, B. L. Foster, M. S. Beauchamp, and D. Yoshor. Saturation in Phosphene Size with Increasing Current Levels Delivered to Human Visual Cortex. J. Neurosci., 37(30):7188-7197, 072017.

[8] William Bosking, Brett Foster, Ping Sun, Mike Beauchamp, and Daniel Yoshor. Rules governing perception of multiple phosphenes by human observers. bioRxiv, page 302547, 2018.

[9] William H Bosking, Ping Sun, Muge Ozker, Xiaomei Pei, Brett L Foster, Michael S Beauchamp, and Daniel Yoshor. Saturation in phosphene size with increasing current levels delivered to human visual cortex. Journal of Neuroscience, 37(30):7188-7197, 2017.

[10] David C Bradley, Philip R Troyk, Joshua A Berg, Martin Bak, Stuart Cogan, Robert Erickson, Conrad Kufta, Massimo Mascaro, Douglas McCreery, Edward M Schmidt, et al. Visuotopic mapping through a multichannel stimulating implant in primate v1. Journal of neurophysiology, 93(3):1659-1670, 2005.

[11] Giles S Brindley and WS Lewin. The sensations produced by electrical stimulation of the visual cortex. The Journal of physiology, 196(2):479-493, 1968.

[12] GS Brindley. The umber of information channels needed for efficient reading. In Journal of Physiology - London, volume 177, page P44, 1965.

[13] GS Brindley. Effects of electrical stimulation of the visual cortex. Hum Neurobiol, 1:281-283, 1982.

[14] Emma Brunton, Arthur J. Lowery, and Ramesh Rajan. A comparison of microelectrodes for a visual cortical prosthesis using finite element analysis. Frontiers in Neuroengineering, $5,2012$.

[15] Christopher R Butson and Cameron C McIntyre. Role of electrode design on the volume of tissue activated during deep brain stimulation. Journal of neural engineering, 3(1):1, 2005. 
[16] Hayriye Cagnan, Timothy Denison, Cameron McIntyre, and Peter Brown. Emerging technologies for improved deep brain stimulation. Nature Biotechnology, 37(9):1024-1033, September 2019.

[17] Giada Cellot, Paola Lagonegro, Giuseppe Tarabella, Denis Scaini, Filippo Fabbri, Salvatore Iannotta, Maurizio Prato, Giancarlo Salviati, and Laura Ballerini. PEDOT:PSS interfaces support the development of neuronal synaptic networks with reduced neuroglia response in vitro. Frontiers in Neuroscience, 9, January 2016.

[18] Kichul Cha, Kenneth Horch, and Richard A Normann. Simulation of a phosphene-based visual field: visual acuity in a pixelized vision system. Annals of biomedical engineering, 20(4):439-449, 1992.

[19] SC Chen, LE Hallum, NH Lovell, and Gregg Jorgen Suaning. Visual acuity measurement of prosthetic vision: a virtual-reality simulation study. Journal of Neural Engineering, 2(1):S135, 2005.

[20] Spencer C Chen, Gregg J Suaning, John W Morley, and Nigel H Lovell. Simulating prosthetic vision: I. visual models of phosphenes. Vision research, 49(12):1493-1506, 2009.

[21] Spencer C Chen, Gregg J Suaning, John W Morley, and Nigel H Lovell. Simulating prosthetic vision: Ii. measuring functional capacity. Vision research, 49(19):2329-2343, 2009.

[22] S. F. Cogan. Neural stimulation and recording electrodes. Annu Rev Biomed Eng, 10:275-309, 2008.

[23] Stuart Cogan, Seth Hara, and Kip Ludwig. The Safe Delivery of Electrical Currents and Neuromodulation, pages 83-94. 012018.

[24] Ethan D Cohen. Prosthetic interfaces with the visual system: biological issues. Journal of neural engineering, 4(2):R14, 2007.

[25] Nathan G Congdon, David S Friedman, and Thomas Lietman. Important causes of visual impairment in the world today. Jama, 290(15):2057-2060, 2003.

[26] X. T. Cui and D. D. Zhou. Poly (3,4-ethylenedioxythiophene) for chronic neural stimulation. IEEE Trans Neural Syst Rehabil Eng, 15(4):502-508, Dec 2007.

[27] Edgar A DeYoe, Jeffrey D Lewine, and Robert W Doty. Laminar variation in threshold for detection of electrical excitation of striate cortex by macaques. Journal of neurophysiology, $94(5): 3443-3450,2005$.

[28] WH Dobelle and MG Mladejovsky. Phosphenes produced by electrical stimulation of human occipital cortex, and their application to the development of a prosthesis for the blind. The Journal of physiology, 243(2):553-576, 1974.

[29] William H Dobelle, MG Mladejovsky, and JP Girvin. Artificial vision for the blind: electrical stimulation of visual cortex offers hope for a functional prosthesis. Science, 183(4123):440 444, 1974.

[30] Wm H Dobelle. Artificial vision for the blind by connecting a television camera to the visual cortex. ASAIO journal, 46(1):3-9, 2000.

[31] Wm H Dobelle, Michael G Mladejovsky, Jerald R Evans, TS Roberts, and JP Girvin. "braille" reading by a blind volunteer by visual cortex stimulation. Nature, 259(5539):111, 1976. 
[32] A. M. Dymond. Characteristics of the metal-tissue interface of stimulation electrodes. IEEE Trans Biomed Eng, 23(4):274-280, Jul 1976.

[33] D.J. Edell, V.V. Toi, V.M. McNeil, and L.D. Clark. Factors influencing the biocompatibility of insertable silicon microshafts in cerebral cortex. IEEE Transactions on Biomedical Engineering, 39(6):635-643, June 1992.

[34] Alexander Farnum and Galit Pelled. New vision for visual prostheses. Frontiers in Neuroscience, 14:36, 2020.

[35] Eduardo Fernández, Arantxa Alfaro, and Pablo González-López. Toward long-term communication with the brain in the blind by intracortical stimulation: Challenges and future prospects. Frontiers in Neuroscience, 14, 2020.

[36] Eduardo Fernández and Richard A Normann. Cortivis approach for an intracortical visual prostheses. In Artificial Vision, pages 191-201. Springer, 2017.

[37] Eduardo Fernandez, Cristina Soto, Arantxa Alfaro, Pablo Gonzalez, Antonio Lozano, Sebastian Pena, Maria Dolores Grima, Alfonso Rodil, Antonio Alarcon, John Rolston, et al. Development of a cortical visual neuroprosthesis for the blind: preliminary results. Investigative Ophthalmology \&6 Visual Science, 60(9):4021-4021, 2019.

[38] Otfrid Foerster. The cerebral cortex in man. Lancet, 2:309-312, 1931.

[39] Shelley Fried and Seung Woo Lee. Abstract\# 39: Towards a microcoil-based cortical visual prosthesis. Brain Stimulation: Basic, Translational, and Clinical Research in Neuromodulation, 12(2):e14, 2019.

[40] Lin Fu, Shanqing Cai, Hui Zhang, Guangshu Hu, and Xudong Zhang. Psychophysics of reading with a limited number of pixels: towards the rehabilitation of reading ability with visual prosthesis. Vision Research, 46(8-9):1292-1301, 2006.

[41] Warren M Grill and J Thomas Mortimer. Electrical properties of implant encapsulation tissue. Annals of biomedical engineering, 22(1):23-33, 1994.

[42] Warren M Grill and J Thomas Mortimer. Stimulus waveforms for selective neural stimulation. IEEE Engineering in Medicine and Biology Magazine, 14(4):375-385, 1995.

[43] Ileana Hancu, Alexandre Boutet, Eric Fiveland, Manish Ranjan, Julia Prusik, Marisa Dimarzio, Tanweer Rashid, Jeffrey Ashe, David Xu, Suneil K Kalia, et al. On the (non-) equivalency of monopolar and bipolar settings for deep brain stimulation fmri studies of parkinson's disease patients. Journal of Magnetic Resonance Imaging, 49(6):1736-1749, 2019.

[44] Jessica L Irons, Tamara Gradden, Angel Zhang, Xuming He, Nick Barnes, Adele F Scott, and Elinor McKone. Face identity recognition in simulated prosthetic vision is poorer than previously reported and can be improved by caricaturing. Vision research, 137:61-79, 2017.

[45] E Jankowska and DO Smith. Antidromic activation of renshaw cells and their axonal projections. Acta Physiologica Scandinavica, 88(2):198-214, 1973.

[46] Thomas Kammer. Phosphenes and transient scotomas induced by magnetic stimulation of the occipital lobe: their topographic relationship. Neuropsychologia, 37(2):191-198, 1998.

[47] Daryl R Kipke, William Shain, György Buzsáki, Eberhard Fetz, Jaimie M Henderson, Jamille F Hetke, and Gerwin Schalk. Advanced neurotechnologies for chronic neural interfaces: new horizons and clinical opportunities. Journal of Neuroscience, 28(46):11830 $11838,2008$. 
[48] Steven Kotler. Vision quest a half century of artificial-sight research has succeeded. and now this blind man can see. behind the bionic-eye breakthrough. WIRED-SAN FRANCISCO-, 10(9):94-94, 2002.

[49] Takashi DY Kozai, Andrea S Jaquins-Gerstl, Alberto L Vazquez, Adrian C Michael, and $\mathrm{X}$ Tracy Cui. Brain tissue responses to neural implants impact signal sensitivity and intervention strategies. ACS chemical neuroscience, 6(1):48-67, 2015.

[50] Doe Kumsa, Eric M Hudak, Fred W Montague, Shawn C Kelley, Darrel F Untereker, Benjamin P Hahn, Chris Condit, Martin Cholette, Hyowon Lee, Dawn Bardot, et al. Electrical neurostimulation with imbalanced waveform mitigates dissolution of platinum electrodes. Journal of neural engineering, 13(5):054001, 2016.

[51] Seung Woo Lee, Florian Fallegger, Bernard DF Casse, and Shelley I Fried. Implantable microcoils for intracortical magnetic stimulation. Science advances, 2(12), 2016.

[52] C. Lettieri, S. Rinaldo, G. Devigili, F. Pisa, M. Mucchiut, E. Belgrado, M. Mondani, S. D'Auria, T. Ius, M. Skrap, and R. Eleopra. Clinical outcome of deep brain stimulation for dystonia: constant-current or constant-voltage stimulation? A non-randomized study. Eur. J. Neurol., 22(6):919-926, Jun 2015.

[53] Xindong Liu, Douglas B McCreery, Leo A Bullara, and William F Agnew. Evaluation of the stability of intracortical microelectrode arrays. IEEE Transactions on Neural Systems and Rehabilitation Engineering, 14(1):91-100, 2006.

[54] Xindong Liu, Douglas B McCreery, Randy R Carter, Leo A Bullara, Ted GH Yuen, and William F Agnew. Stability of the interface between neural tissue and chronically implanted intracortical microelectrodes. IEEE transactions on rehabilitation engineering, 7(3):315-326, 1999.

[55] Arthur James Lowery, Jeffrey V Rosenfeld, Marcello GP Rosa, Emma Brunton, Ramesh Rajan, Collette Mann, Mark Armstrong, Anand Mohan, Horace Josh, Lindsay Kleeman, et al. Monash vision group's gennaris cortical implant for vision restoration. In Artificial Vision, pages 215-225. Springer, 2017.

[56] Yichen Lu, Hongming Lyu, Andrew G Richardson, Timothy H Lucas, and Duygu Kuzum. Flexible neural electrode array based-on porous graphene for cortical microstimulation and sensing. Scientific reports, 6, 2016.

[57] Jaakko Malmivuo and Robert Plonsey. Bioelectromagnetism - Principles and Applications of Bioelectric and Biomagnetic Fields. 031995.

[58] Elwin Marg and David Rudiak. Phosphenes induced by magnetic stimulation over the occipital brain: description and probable site of stimulation. Optometry and vision science: official publication of the American Academy of Optometry, 71(5):301-311, 1994.

[59] Cameron C McIntyre and Warren M Grill. Selective microstimulation of central nervous system neurons. Annals of biomedical engineering, 28(3):219-233, 2000.

[60] D. R. Merrill, M. Bikson, and J. G. Jefferys. Electrical stimulation of excitable tissue: design of efficacious and safe protocols. J. Neurosci. Methods, 141(2):171-198, Feb 2005.

[61] W. Alan Mutch and Anker Hansen. Brain Extracellular pH Changes During Alterations in Substrate Supply, pages 189-193. 011985.

[62] Soroush Niketeghad, Abirami Muralidharan, Uday Patel, Jessy D Dorn, Laura Bonelli, Robert J Greenberg, and Nader Pouratian. Phosphene perceptions and safety of chronic visual cortex stimulation in a blind subject. Journal of neurosurgery, 132(6):2000-2007, 2019 . 
[63] Soroush Niketeghad and Nader Pouratian. Brain machine interfaces for vision restoration: The current state of cortical visual prosthetics. Neurotherapeutics, 16, 092018.

[64] Soroush Niketeghad and Nader Pouratian. Brain machine interfaces for vision restoration: the current state of cortical visual prosthetics. Neurotherapeutics, 16(1):134-143, 2019.

[65] Richard A Normann, Edwin M Maynard, Patrick J Rousche, and David J Warren. A neural interface for a cortical vision prosthesis. Vision research, 39(15):2577-2587, 1999.

[66] Richard A Normann, David Warren, and Alexei Koulakov. Representations and dynamics of representations of simple visual stimuli by ensembles of neurons in cat visual cortex studied with a microelectrode array. In Neural Engineering, 2003. Conference Proceedings. First International IEEE EMBS Conference on, pages 91-94. IEEE, 2003.

[67] World Health Organization. World report on vision. Geneva, Switzerland, World Health Organization, 2019.

[68] Hyunsu Park, Pavel Takmakov, and Hyowon Lee. Electrochemical evaluations of fractal microelectrodes for energy efficient neurostimulation. Scientific Reports, 8(1), March 2018.

[69] Michael Polanco, Sebastian Bawab, and Hargsoon Yoon. Computational assessment of neural probe and brain tissue interface under transient motion. Biosensors, 6(2):27, 2016.

[70] DA Pollen. Some perceptual effects of electrical stimulation of the visual cortex in man. The nervous system, 2:519-528, 1975.

[71] Daniel A Pollen. Responses of single neurons to electrical stimulation of the surface of the visual cortex. Brain, behavior and evolution, 14(1-2):67-86, 1977.

[72] Kelsey A Potter, Amy C Buck, Wade K Self, and Jeffrey R Capadona. Stab injury and device implantation within the brain results in inversely multiphasic neuroinflammatory and neurodegenerative responses. Journal of neural engineering, 9(4):046020, 2012.

[73] Anmona S. Pranti, Andreas Schander, André Bödecker, and Walter Lang. PEDOT: PSS coating on gold microelectrodes with excellent stability and high charge injection capacity for chronic neural interfaces. Sensors and Actuators B: Chemical, 275:382-393, December 2018.

[74] J. V. Raimondo, R. J. Burman, A. A. Katz, and C. J. Akerman. Ion dynamics during seizures. Front Cell Neurosci, 9:419, 2015.

[75] James B Ranck Jr. Which elements are excited in electrical stimulation of mammalian central nervous system: a review. Brain research, 98(3):417-440, 1975.

[76] R. Ranjandish and A. Schmid. A compact size charge-mode stimulator using a low-power active charge balancing method for deep brain stimulation (dbs). In 2017 IEEE Biomedical Circuits and Systems Conference (BioCAS), pages 1-4, 2017.

[77] Reza Ranjandish, Omid Shoaei, and Alexandre Schmid. A fully fail-safe capacitive-based charge metering method for active charge balancing in deep brain stimulation. 2018 14th Conference on Ph.D. Research in Microelectronics and Electronics (PRIME), pages 249-252, 2018.

[78] Patrick J Rousche, David S Pellinen, David P Pivin, Justin C Williams, Rio J Vetter, and Daryl R Kipke. Flexible polyimide-based intracortical electrode arrays with bioactive capability. IEEE Transactions on biomedical engineering, 48(3):361-371, 2001. 
[79] Alexander D Rush and Philip R Troyk. A power and data link for a wireless-implanted neural recording system. IEEE Transactions on Biomedical Engineering, 59(11):3255-3262, 2012.

[80] DN Rushton and GS Brindley. Short-and long-term stability of cortical electrical phosphenes. Physiological aspects of clinical neurology, pages 123-153, 1977.

[81] Mesut Sahin and Yanmei Tie. Non-rectangular waveforms for neural stimulation with practical electrodes. Journal of neural engineering, 4(3):227, 2007.

[82] Joseph W Salatino, Kip A Ludwig, Takashi DY Kozai, and Erin K Purcell. Glial responses to implanted electrodes in the brain. Nature biomedical engineering, 1(11):862-877, 2017.

[83] Peter H Schiller and Edward J Tehovnik. Visual prosthesis. Perception, 37(10):1529-1559, 2008.

[84] EM Schmidt, MJ Bak, FT Hambrecht, CV Kufta, DK O'rourke, and P Vallabhanath. Feasibility of a visual prosthesis for the blind based on intracortical micro stimulation of the visual cortex. Brain, 119(2):507-522, 1996.

[85] Robert V Shannon. A model of safe levels for electrical stimulation. IEEE Transactions on biomedical engineering, 39(4):424-426, 1992.

[86] Jörg Sommerhalder, Evelyne Oueghlani, Marc Bagnoud, Ute Leonards, Avinoam B Safran, and Marco Pelizzone. Simulation of artificial vision: I. eccentric reading of isolated words, and perceptual learning. Vision Research, 43(3):269-283, 2003.

[87] Paul Stark, Giovanni Fazio, and Eugene S Boyd. Monopolar and bipolar stimulation of the brain. American Journal of Physiology-Legacy Content, 203(2):371-373, 1962.

[88] Edward J Tehovnik. Electrical stimulation of neural tissue to evoke behavioral responses. Journal of neuroscience methods, 65(1):1-17, 1996.

[89] Edward J Tehovnik, Warren M Slocum, Christina E Carvey, and Peter H Schiller. Phosphene induction and the generation of saccadic eye movements by striate cortex. Journal of neurophysiology, 93(1):1-19, 2005.

[90] Edward J Tehovnik, Warren M Slocum, and Peter H Schiller. Saccadic eye movements evoked by microstimulation of striate cortex. European Journal of Neuroscience, 17(4):870-878, 2003.

[91] Edward J Tehovnik, Warren M Slocum, and Peter H Schiller. Microstimulation of v1 delays the execution of visually guided saccades. European Journal of Neuroscience, 20(1):264-272, 2004.

[92] Edward J Tehovnik, Warren M Slocum, and Peter H Schiller. Delaying visually guided saccades by microstimulation of macaque v1: spatial properties of delay fields. European Journal of Neuroscience, 22(10):2635-2643, 2005.

[93] Edward J Tehovnik, Warren M Slocum, Stelios M Smirnakis, and Andreas S Tolias. Microstimulation of visual cortex to restore vision. Progress in brain research, 175:347-375, 2009.

[94] Margaret C Thompson, Jeffrey A Herron, Timothy Brown, Jeffrey G Ojemann, Andrew L Ko, and Howard J Chizeck. Demonstration of a stable chronic electrocorticography-based brain-computer interface using a deep brain stimulator. In 2016 IEEE International Conference on Systems, Man, and Cybernetics (SMC), pages 002936-002941. IEEE, 2016. 
[95] Vernon L Towle, Peter Pytel, Frank Lane, John Plass, David M Frim, and Philip R Troyk. Postmortem investigation of a human cortical visual prosthesis that was implanted for 36 years. Journal of Neural Engineering, 17(4):045010, 2020.

[96] Philip Troyk, Martin Bak, Joshua Berg, David Bradley, Stuart Cogan, Robert Erickson, Conrad Kufta, Douglas McCreery, Edward Schmidt, and Vernon Towle. A model for intracortical visual prosthesis research. Artificial organs, 27(11):1005-1015, 2003.

[97] PR Troyk, DEA Detlefsen, and GAD DeMichele. A multifunctional neural electrode stimulation asic using neurotalk tm interface. In Engineering in Medicine and Biology Society, 2006. EMBS'06. 28th Annual International Conference of the IEEE, pages 29942997. IEEE, 2006.

[98] Joram J van Rheede, Christopher Kennard, and Stephen L Hicks. Simulating prosthetic vision: Optimizing the information content of a limited visual display. Journal of vision, $10(14): 32-32,2010$.

[99] Jonathan Viventi, Dae-Hyeong Kim, Leif Vigeland, Eric S Frechette, Justin A Blanco, Yun-Soung Kim, Andrew E Avrin, Vineet R Tiruvadi, Suk-Won Hwang, Ann C Vanleer, et al. Flexible, foldable, actively multiplexed, high-density electrode array for mapping brain activity in vivo. Nature neuroscience, 14(12):1599, 2011.

[100] Milena Vurro, Giuseppe Baselli, Francesco Orabona, and Giulio Sandini. Simulation and assessment of bioinspired visual processing system for epi-retinal prostheses. In Engineering in Medicine and Biology Society, 2006. EMBS'06. 28th Annual International Conference of the IEEE, pages 3278-3281. IEEE, 2006.

[101] Brian A Wandell, Serge O Dumoulin, and Alyssa A Brewer. Visual field maps in human cortex. Neuron, 56(2):366-383, 2007.

[102] David J Warren and Richard A Normann. Functional reorganization of primary visual cortex induced by electrical stimulation in the cat. Vision research, 45(5):551-565, 2005.

[103] Xuefeng F Wei and Warren M Grill. Current density distributions, field distributions and impedance analysis of segmented deep brain stimulation electrodes. Journal of neural engineering, 2(4):139, 2005.

[104] Xuefeng F Wei and Warren M Grill. Analysis of high-perimeter planar electrodes for efficient neural stimulation. Frontiers in neuroengineering, 2:15, 2009.

[105] James D Weiland and David J Anderson. Chronic neural stimulation with thin-film, iridium oxide electrodes. IEEE Transactions on Biomedical Engineering, 47(7):911-918, 2000.

[106] Jonathan Winawer and Josef Parvizi. Linking electrical stimulation of human primary visual cortex, size of affected cortical area, neuronal responses, and subjective experience. Neuron, 92(6):1213-1219, 2016.

[107] Ying Zhao, Xiulin Geng, Qi Li, Guangqi Jiang, Yu Gu, and Xiaoqi Lv. Recognition of a virtual scene via simulated prosthetic vision. Frontiers in bioengineering and biotechnology, $5: 58,2017$. 